\title{
A comparison of methods of discontinuing nasal CPAP in premature infants $<30$ weeks gestation: a feasibility study
}

\author{
Brittany Duyka iD ${ }^{1 凶}$, Claire Beaullieu ${ }^{1}$ and Amir M. Khan ${ }^{1}$ \\ (c) The Author(s), under exclusive licence to Springer Nature America, Inc. 2021
}

\begin{abstract}
OBJECTIVE: The objective of this study was to determine whether abrupt discontinuation vs gradual wean of nasal CPAP (NCPAP) in infants $<30$ weeks gestation results in a decreased duration of NCPAP therapy.

STUDY DESIGN: We performed a single-center, randomized control trial of premature infants born $<30$ weeks gestation $(n=66)$, comparing discontinuation of NCPAP from $6 \mathrm{cmH}_{2} \mathrm{O}$ (CWP) (fast wean arm) to discontinuation at 4 CWP, weaning by 1 CWP per day (slow wean arm). The primary outcome was the total number of days on NCPAP or mechanical ventilation. Secondary outcomes included wean failure, growth, length of stay, and related comorbidities.

RESULTS: Duration of NCPAP or mechanical ventilation was longer in the slow wean arm compared to the fast wean arm (17 vs 12 days, $p=0.03$ ). There were no differences observed in secondary outcomes.

CONCLUSION: In weaning NCPAP, abrupt discontinuation may be associated with a shorter duration of positive pressure respiratory support compared to a gradual weaning strategy.
\end{abstract}

Journal of Perinatology (2021) 41:2658-2663; https://doi.org/10.1038/s41372-021-01200-9

\section{BACKGROUND}

Nasal continuous positive airway pressure (NCPAP) is currently widely used to provide noninvasive respiratory support for premature infants, especially those who are $<30$ weeks of gestation [1, 2]. Recently, three large randomized controlled trials have shown that NCPAP therapy initiated in the delivery room is equally effective as the prophylactic administration of surfactants in the treatment of respiratory distress syndrome [3-5]. In addition, there are data to suggest that volutrauma and atelectrauma from mechanical ventilation play an important role in the development of bronchopulmonary dysplasia (BPD) [6]. NCPAP is effective in lung recruitment and improving functional residual capacity while avoiding the trauma associated with invasive mechanical ventilation [7]. Therefore, premature infants are preferentially treated with NCPAP rather than mechanical ventilation, to reduce the risk of BPD. However, while there is a significant body of literature justifying the use of NCPAP in premature infants, very little is known about its management, such as how long to continue NCPAP and, more importantly, how and when to wean off NCPAP. Data regarding how and when to take a patient off NCPAP remains inconclusive. Strategies that have been studied include gradual vs abrupt weaning of pressures, cycling on and off NCPAP with a stepwise increase in time off NCPAP until discontinuation, and weaning to high flow nasal cannula (HFNC) as an intermediary step between NCPAP and nasal cannula. There are currently five published trials, the first by Abdel-Hady et al. in 1998 that showed infants are more likely to tolerate discontinuation of NCPAP after a gradual reduction in pressure with some increase in oxygen requirement [8]. The second trial by the same authors in 2011 showed that weaning from NCPAP to supplemental oxygen via HFNC did not decrease the duration of NCPAP therapy, but increased the length of oxygen use [9]. Another recent trial by Todd et al. in 2012 showed that discontinuation of NCPAP, rather than cycling NCPAP, resulted in earlier discontinuation of NCPAP [10]. There have been two recent studies by Amatya et al. and Jensen et al. looking at the primary weaning strategies of abrupt vs gradual wean [1114]. These studies did not find a difference in the outcomes of NCPAP duration and time to successful weaning from NCPAP. In the clinical setting, practice varies from discontinuation of CPAP when the infant is stable or gradually reducing pressures before discontinuation based on provider preferences. Currently, there is no clear evidence that either method of weaning is more effective in reducing the duration of NCPAP therapy. Each day on NCPAP is associated with potential clinical risks (i.e., nasal irritation/breakdown, abdominal distension), patient discomfort, utilization of resources, and time-intensive, meticulous workload for staff. In light of this, we concluded that the number of days on NCPAP would be the most pragmatic primary outcome in our investigation. We sought to answer this question by conducting a randomized trial of discontinuing NCPAP with or without weaning off the pressure and assess the effect of each strategy on the number of days on NCPAP or mechanical ventilation. Our study question was as follows: In premature infants $<30$ weeks gestation who are stable on NCPAP, does abrupt discontinuation of NCPAP compared to gradual wean of NCPAP prior to discontinuation result in few total number of days on NCPAP or mechanical ventilation in a 28-day period?

${ }^{1}$ McGovern Medical School, University of Texas Health Science Center, Houston, TX, USA. ${ }^{凶}$ email: blduyka@gmail.com 


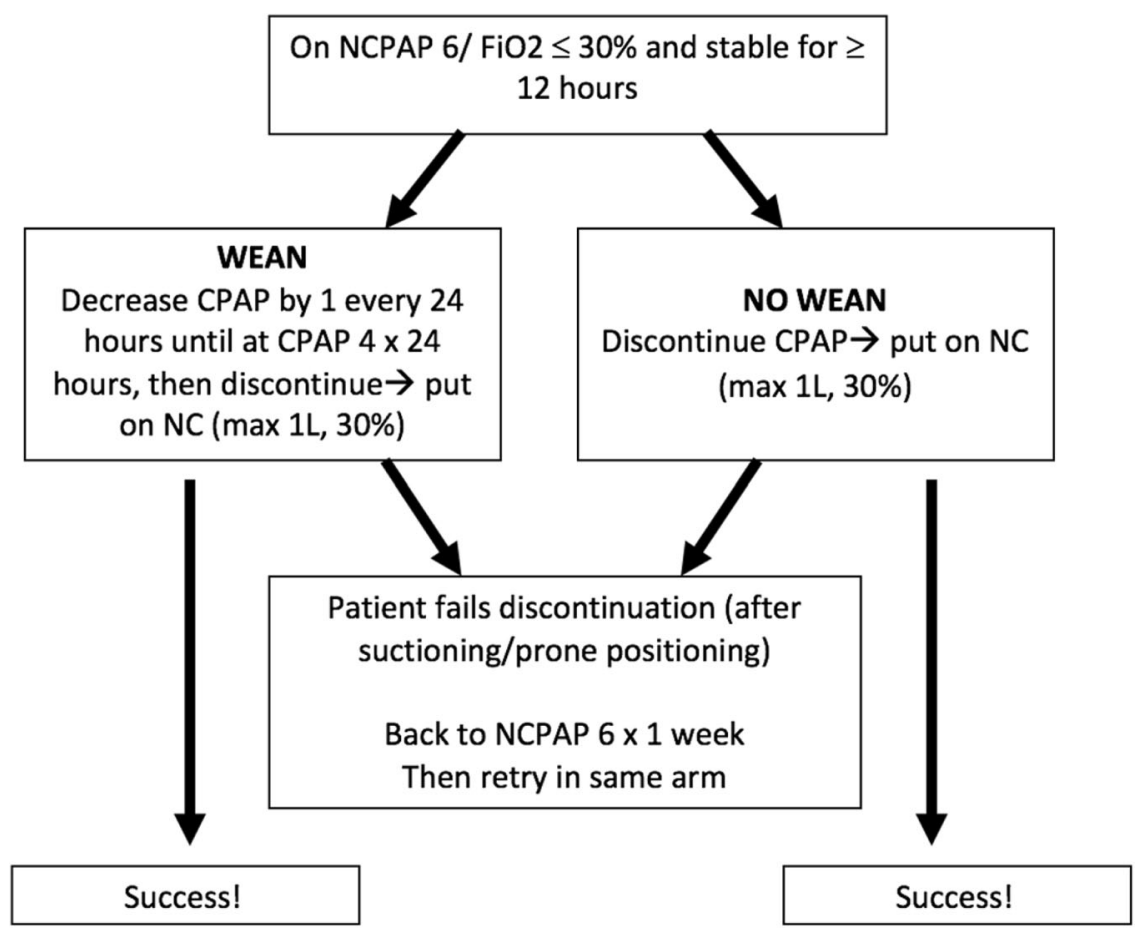

Fig. 1 Study algorithm. Resource placed at bedside and in NICU work areas to guide NCPAP management for patients enrolled in the study.

\section{METHODS}

We conducted a randomized controlled, comparative effectiveness trial in a large academic Level IV neonatal intensive care unit in Houston, Texas, between August 2017 and March 2019. The Institutional Review Board at the University of Texas Health Science Center at Houston approved the study before implementation, and the trial was registered at clinicaltrials. gov (NCT03292562).

We included all neonates $<30$ weeks gestational age at birth who were on NCPAP $6 \mathrm{cmH}_{2} \mathrm{O}$ (CWP), on treatment for apnea of prematurity with caffeine $10 \mathrm{mg} / \mathrm{kg}$ daily, and met the stability criteria for at least $12 \mathrm{~h}$. The stability criteria were prespecified and similar to previous studies, particularly the study by Todd et al. in 2012 [10], to minimize provider variability. These stability criteria included all of the following: NCPAP 6 CWP requiring $\mathrm{FiO}_{2}$ between 0.21 and 0.25 to maintain oxygen saturations $85-95 \%$ (consistent with the unit target for oxygen saturations); respiratory rate consistently $1<60$ breaths per minute, mild to no subcostal/intercostal retractions, no apnea or bradycardia events requiring bag-mask ventilation in the past $12 \mathrm{~h},<3$ apnea and/or bradycardic episodes in any 1 -h period for the previous $6 \mathrm{~h}$, and tolerating time off CPAP during routine CPAP care ( $15 \mathrm{~min})$. Parental consent was obtained in the first week of life or the first week of admission to our hospital if transferred from an outside facility. We identified potential study patients by daily census review and approached the parents of all infants born $<30$ weeks who were on NCPAP or mechanical ventilation for consent.

Exclusion criteria included major congenital anomalies (including congenital heart disease), conditions that prevented discontinuation of NCPAP such as severe tracheomalacia, and patients who were undergoing evaluation and/or treatment for sepsis at the time of enrollment.

Consented patients were randomized once predefined stability criteria were met on NCPAP 6 CWP and the provider was ready to discontinue or wean NCPAP. A randomization allocation sequence was created using permuted blocks in a 4-6-6 scheme. The study group assignment was placed in sequentially numbered, opaque envelopes. The primary medical team obtained the subsequently numbered randomization envelope and followed the assigned intervention protocol. The primary medical team had no influence on what type of envelope was chosen. Blinding providers to the intervention group was not feasible given the nature of workflow in our unit. In order to minimize provider variability and keep the practice as consistent as possible, the protocols did not allow for the use of HFNC or flow $>1 \mathrm{l} / \mathrm{min}$ (LPM) as an intermediary step between NCPAP and nasal cannula. Other than the requirement for enrolled infants to be on caffeine $10 \mathrm{mg} / \mathrm{kg}$ daily at the time of randomization, the adjustment and discontinuation of caffeine were at the provider's discretion.

\section{Interventions (Fig. 1)}

Fast wean arm. Infants who were randomized to the abrupt discontinuation or "fast wean" group had NCPAP discontinued from 6 CWP and were placed on a nasal cannula, 1 LPM on no $>30 \%$ supplemental oxygen. Infants were considered to have failed discontinuation if one or more of the following criteria were met: persistent respiratory rate $>75$ breaths per minute; respiratory distress defined as moderate to severe retractions, grunting, nasal flaring that persisted for at least $30 \mathrm{~min} ; \mathrm{FiO}_{2}>30 \%$ for at least $6 \mathrm{~h}$ to keep oxygen saturations $85-95 \%$; any apnea or bradycardia that required bag-mask ventilation, or more than two apnea and/or bradycardia episodes in a 1-h period. If an infant failed discontinuation, NCPAP of at least 6 CWP was resumed for a minimum of 1 week before another attempt to discontinue NCPAP was made, after the patient again met the stability criteria. If an infant met the failure criteria after the second attempt, the study ended for that patient and respiratory support was managed at the providers' discretion.

Slow wean arm. Infants who were randomized to the slow wean group had CPAP pressure weaned from 6 CWP by 1 CWP every $24 \mathrm{~h}$ if the subjects met the stability criteria after each wean until reaching CPAP 4 CWP. If at any point after decreasing CPAP pressure the subject met CPAP failure criteria, the pressure was increased back to the previous CPAP pressure. After achieving stabilization for $24 \mathrm{~h}$, the weaning process was resumed again. Once the subject met the stability criteria on CPAP of 4 CWP for at least $24 \mathrm{~h}$, NCPAP was discontinued, and the infant was placed on nasal cannula at 1 LPM and no $>30 \%$ supplemental oxygen. If the infant failed discontinuation, they were put back on NCPAP 6 CWP for a minimum of 1 week before attempting to wean the NCPAP, as long as the patient met the stability criteria. If an infant met the failure criteria after the second attempt, the study ended for that patient and respiratory support was managed at the providers' discretion.

\section{Outcomes}

The study period ended 28 days after randomization or after two failed attempts of discontinuation of NCPAP, whichever occurred earlier. The primary outcome of interest was the total number of days on NCPAP or mechanical ventilation from randomization until discharge. Secondary outcomes included failure to wean off NCPAP within 28 days or two failed attempts of discontinuation, success at first weaning attempt, time to successful wean, postmenstrual age and weight at successful wean and at discharge, time to ad lib feeding by mouth, and length of hospital stay. We also investigated rates of comorbidities that occurred after randomization, 


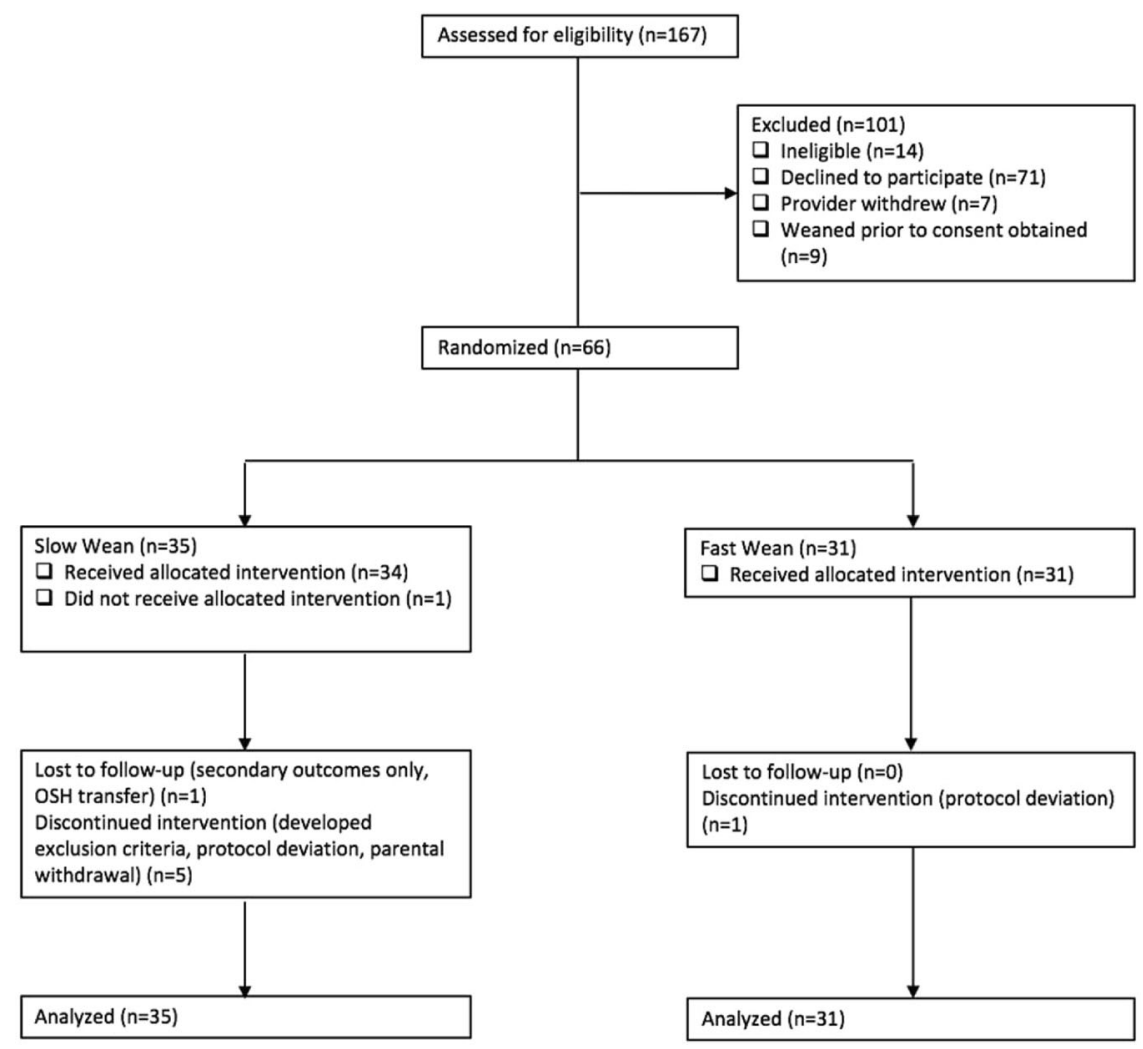

Fig. 2 Study enrollment flow chart and participant retention. Study enrollment flow diagram.

including BPD, necrotizing enterocolitis, air leak disorders, nasal breakdown, and the need for home oxygen therapy after discharge. Since providers were free to use HFNC once the patient was no longer in the study, we also investigated the number of days on HFNC during the 28-day study period in infants who failed two attempts in NCPAP discontinuation.

\section{Statistical analysis}

Prior to the initiation of our study, no published studies evaluated our primary outcome with a similar weaning methodology, which limited our ability to calculate the appropriate sample size. Therefore, we designed our study as a pilot study for a larger, more definitive study in the future. Since no sample size was calculated, we planned as many patients as possible within 1 year, ideally achieving a similar sample size to the studies comparing other methods of weaning CPAP. Data were analyzed with the intention-to-treat principle using the STATA statistical analysis software. Continuous variables were analyzed using the Student's $t$ test and nominal variables were analyzed using the $x^{2}$ test. A time-to-success analysis was done using the Kaplan-Meier survival analysis. Time to success was defined as the number of days from the day the patient was randomized until the day that patient was taken off NCPAP without failure, within the 28-day study period. Those who failed discontinuation of NCPAP twice or who were not weaned off NCPAP during the 28-day study period were censored out. The hazard ratio was calculated using the Cox regression.

\section{RESULTS}

One hundred and sixty-seven potential study participants were admitted during the enrollment period of August 2017 to November 2018. Of these, seven (4\%) patients were ineligible (one was critically ill and had do not resuscitate in place, four died prior to consent being obtained, two had major congenital anomalies). Nine (5\%) patients had NCPAP weaned prior to consent being obtained. Parents were approached for consent for the remaining 151 patients, and $79(47 \%)$ consented to participate in the study. Of the 79 consented patients, $66(41 \%)$ were enrolled and randomized, as outlined in Fig. 2. Of the thirteen patients who were consented but not randomized, seven were withdrawn from the study by the provider prior to randomization and six were excluded from the study prior to randomization (two died, one developed gastric perforation, one developed severe nasal breakdown, and two were never successfully extubated and required eventual tracheostomy placement). One additional patient was randomized inadvertently without consent in place and was removed from the study after relevant notifications. Thirty-five infants were randomized to the "slow wean" arm, while 31 infants were randomized to the "fast wean" arm. One patient was randomized to the "slow wean" arm, but the provider inadvertently followed the "fast wean" protocol and was analyzed with the "slow wean" arm based on the intention-totreat principle. The clinical characteristics were similar in each group except for antenatal steroid treatment, which was less in the "slow wean" group (Table 1).

Infants in the "fast wean" group were on NCPAP or mechanical ventilation for an average of 12.3 days (95\% confidence interval (CI) 8.9-15.6), while infants in the "slow wean" group were on NCPAP or mechanical ventilation for an average of 17.3 days $(95 \%$ $\mathrm{Cl}$ 14.2-20.3) (Table 2). The difference between the means was -5 , which was statistically significant with $95 \% \mathrm{Cl}$ of -9.5 to -0.5 and $p$ value of 0.029 . There were no differences observed in any of the secondary outcomes measured. We chose to present the data in Table 2 as means with $95 \% \mathrm{Cls}$ instead of standard deviations in order to provide a more meaningful inference of the results by showing precision of estimates as well as the potential range of effects seen in the context of the sample variability. A per-protocol analysis was also performed comparing the 34 patients in the "slow wean" arm and the 32 patients in the "fast wean" arm. The per-protocol analysis, like the intention-to-treat analysis, showed that the infants who followed the "fast wean" protocol were on NCPAP or mechanical ventilation 5.4 fewer days than those in the "slow wean" group $(17.5$ days $(95 \% \mathrm{Cl} 14.3-20.6)$ vs 12.1 days $(95 \%$ Cl 8.9-15.4); $p=0.02$ ).

Kaplan-Meier survival analysis was performed to evaluate time to success in weaning off NCPAP, censored by study failures 
Table 1. Baseline characteristics of study participants.

Characteristics
Sex (M) (\#, \%)
GA at birth (weeks) (mean + range)
Birthweight (g) (mean + range)
PMA at enrollment (weeks) (mean + range)
Weight at enrollment (g) (mean + range)
No antenatal steroids (\#, \%)
Surfactant doses (mean + range)
NCPAP days ${ }^{\mathrm{a}}$ (mean + range)
Ventilator days ${ }^{\mathrm{a}}$ (mean + range)

NIPPV days ${ }^{a}$ (mean + range)

Slow wean $(\boldsymbol{n}=\mathbf{3 5})$
$15(43)$
$26.8(23.7-29.9)$
$965(460-1950)$
$31.4(26.1-42.4)$
$1384(560-3680)$
$9(26 \%)$
$1.17(0-3)$
$14.7(2-58)$
$19.2(0-65)$
$1.5(0-22)$

Fast wean $(n=31)$

12 (38)

$26.8(23.1-29.7)$

$897(500-1480)$

$31.1(27.3-35.2)$

$1237(6400-2235)$

$2(6 \%)$

$1.1(0-3)$

$12.3(2-34)$

$17.6(0-59)$

$1.9(0-18)$

NCPAP nasal continuous positive airway pressure, NIPPV noninvasive positive pressure ventilation, $P M A$ postmenstrual age, GA gestational age.

a Prior to enrollment: NCPAP, ventilator, and NIPPV days were evaluated for each study participant prior to enrollment so that any differences between theoretical risk of lung injury due to positive pressure ventilation prior to randomization could be observed.

(Fig. 3). There was no difference observed between the two groups in time to successful weaning. The median time to success was 19 days in the "fast wean" arm compared to 17 days in the "slow wean" arm. The hazard ratio was 1.44 (95\% Cl 0.7-2.8; $p=$ $0.28)$.

\section{DISCUSSION}

We carried out a randomized, comparative effectiveness trial comparing two commonly used methods of weaning NCPAP and found that gradually weaning NCPAP pressures before discontinuation of NCPAP resulted in infants being on NCPAP for at least 5 days longer than those who had NCPAP abruptly discontinued. Due to the lack of published studies with weaning methods and primary outcomes similar to our study, we designed this study as a pilot study with the expectation that future confirmatory studies would be performed with an appropriate sample size to achieve $80 \%$ power. In contrast to the primary outcomes explored in the previous studies comparing NCPAP weaning methods, we chose to look at the primary outcome of the number of days on NCPAP or mechanical ventilation in a 28-day period as we found this information to be the most clinically and practically relevant. Proper NCPAP use is very staff intensive, requiring frequent, meticulous attention to detail to ensure appropriate positioning and function. It also requires particular care and attention to avoid consequential injury such as nasal breakdown or at worst columellar pressure necrosis. In addition, NCPAP administration can be the determining factor for critical care (higher cost) billing day vs an intensive care (lower cost) billing day. In these cases, unnecessarily prolongation of NCPAP use can result in higher health care costs. It is important in the practice of medicine that we utilize our resources appropriately, seeking the minimum dosage, duration, and cost of treatment to achieve our desired outcome in a safe way.

In our evaluation of secondary outcomes, we observed no significant differences, most notably in the risk for BPD, weight gain, or discharge home on oxygen supplementation. This indicates that either method is likely safe. Interestingly, there was a slight trend toward BPD in the "slow wean" group; however, this was not statistically significant. There were also no incidences of air leak disorders in either group after randomization, and the rates of nasal breakdown were not significantly different in the two groups, indicating that risks of NCPAP therapy were not different in either strategy. While the rates of success at the first attempt were slightly higher in the "slow wean" arm (14 vs 8$)$, this was also not statistically significant $(p=0.22)$. Time to successful weaning off NCPAP was also not different between the two groups, which could indicate that success at first wean attempt is not a good indicator of a successful NCPAP weaning strategy. Interestingly, the secondary outcomes of postmenstrual age at success and weight at success were similar between the two groups (34.1 weeks (28.4-42.1) vs 34.4 weeks (29-52.40), $1847 \mathrm{~g}$ (790-3605) vs $1893 \mathrm{~g}(970-4800)$. This raises the question of whether or not the size and maturity of the infant have more influence on successful discontinuation from NCPAP than the method of weaning. Further studies would need to be done to answer this question definitively.

There have been two recently published studies comparing similar NCPAP weaning methods. The first, by Amatya et al. in 2017, studied infants 26-32 weeks gestation, comparing sudden discontinuation from NCPAP 5 CWP vs a gradual wean from NCPAP 5-3 CWP within 24 h [11] unlike our study where the infant was gradually over several days. They found that their primary outcome of success at the first wean attempt was more likely in those who underwent a gradual wean. They did not see a difference in the total number of days on NCPAP between the two groups, nor did they see a difference in corrected gestational age or weight at successful wean. Most recently, there was a large multicenter randomized controlled trial by Jensen et al. in 2018 that compared a sudden vs gradual pressure wean of NCPAP pressures in infants $<32$ weeks gestation [12]. This study looked at the primary outcome of weight gain velocity and showed no difference between the two groups. Among their secondary outcomes, they also showed no difference in the number of days on NCPAP after randomization, the number of attempts to successful discontinuation of NCPAP, days to successful discontinuation of NCPAP, or length of hospital stay. A stratified analysis did show a higher likelihood of success at first wean attempt in the weaning group in those $<28$ weeks gestation; however, no differences in NCPAP duration in this subgroup.

Our study outcome differed from the other published studies in that we did observe a difference in days on NCPAP and mechanical ventilation after randomization favoring those in the abrupt discontinuation group despite having a small sample size. This will need to be confirmed in future studies. We also did not detect a difference in success at the first wean attempt between the two groups, which differed from similar previous studies. It is possible that our study was not sufficiently powered to detect this difference due to our small sample size.

\section{LIMITATIONS}

The nature of a comparative effectiveness trial lends itself to bias and confounding. We sought to limit provider bias by allocation 
Table 2. Study outcomes.

\begin{tabular}{|c|c|c|c|}
\hline Outcomes & Slow wean $(n=35)$ & Fast wean $(n=31)$ & $P$ value \\
\hline NCPAP/ventilator days (mean, $95 \% \mathrm{Cl}$ ), & $17.3(14.2-20.4)$ & $12.3(8.9-15.6)$ & $\begin{array}{l}0.03 \\
\mathrm{MD}(95 \% \mathrm{Cl})-5(-9.5,-0.5)\end{array}$ \\
\hline Failure (\# of patients, \%) $(95 \% \mathrm{CI})^{\mathrm{c}}$ & $13(37 \%)(21-53 \%)$ & $16(52 \%)(34-70 \%)$ & 0.24 \\
\hline Days to success (mean, $95 \% \mathrm{Cl}$ ) ${ }^{\mathrm{b}, \mathrm{d}}$ & $21.4(16-26.9)$ & $25.3(17.3-33.3)$ & 0.58 \\
\hline PMA at success (weeks) (mean, $95 \% \mathrm{Cl})^{\mathrm{e}}$ & $34.1(33-35.2)$ & $34.4(32.3-36.1)$ & 0.92 \\
\hline PMA at discharge (weeks) (mean, 95\% Cl) & $39.6(38.2-41)$ & $40.1(38.3-41.8)$ & 0.66 \\
\hline Ad lib feeds (DOL) (mean, $95 \% \mathrm{Cl})^{\mathrm{f}}$ & $78(69-88)$ & $73(64-82)$ & 0.45 \\
\hline Weight gain $(\%)(95 \% \mathrm{Cl})^{\mathrm{g}}$ & $196(146-246)$ & $185(144-227)$ & 0.75 \\
\hline BPD (moderate-severe) (\#, \%) $(95 \% \mathrm{Cl})^{\mathrm{h}}$ & $18(51 \%)(36-66 \%)$ & $14(45 \%)(27-63 \%)$ & 0.18 \\
\hline Nasal breakdown (\# of patients, \%) $(95 \% \mathrm{Cl})^{\mathrm{i}}$ & $4(11 \%)(1-21 \%)$ & $2(6 \%)(0-17 \%)$ & 0.48 \\
\hline
\end{tabular}

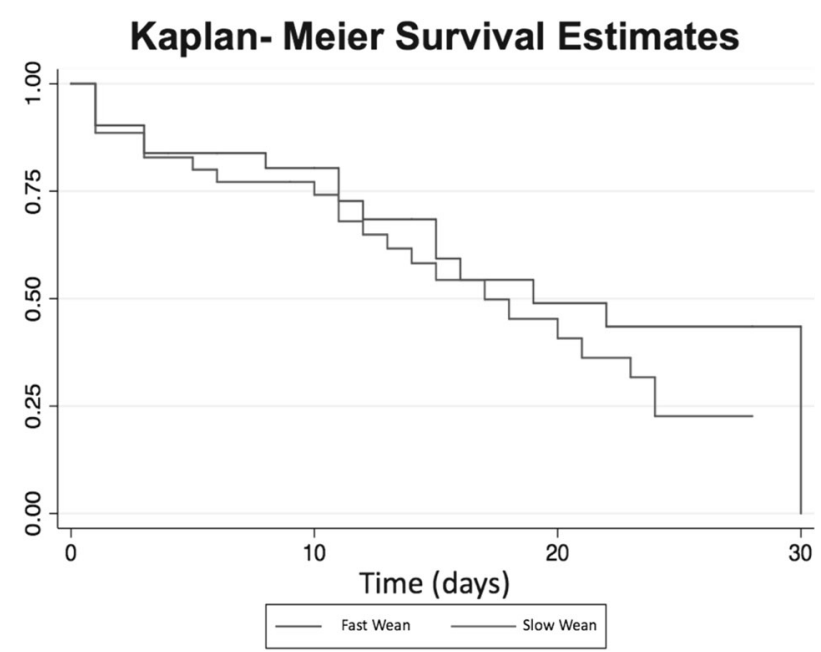

Fig. 3 Kaplan-Meier curves evaluating time to successful weaning, from randomization until successful wean off NCPAP. Censored for failures (defined as failure of NCPAP discontinuation twice or inability to wean from NCPAP during a 28-day study period).

concealment and standardization of stability and failure criteria. In addition, we gave strict study parameters regarding the use of nasal cannula after NCPAP discontinuation and prohibiting the use of HFNC in order to minimize practice variability. We also minimized selection bias by recruiting and enrolling all patients $<30$ weeks gestation who were on mechanical ventilation or NCPAP early in life, prior to considering other factors in their hospital course (such as duration of mechanical ventilation or other factors that could lead to difficulty weaning off NCPAP). While equipoise was present in the study investigators, many providers considered a gradual wean to be a safer, more effective means of weaning NCPAP and the potential for provider bias remained. It is encouraging that despite possible provider bias toward a gradual wean in our unity, the results of our study still favored an abrupt continuation.

Another limitation of our study was the inability to calculate a sample size to ensure adequate power, as it was carried out as a pilot study. While we did not see any significant differences in our secondary outcomes, it is possible that the study was underpowered to detect these differences. Therefore, it is essential that a follow-up study with a sample size calculation to ensure adequate power is performed.

In addition, due to practical constraints, we could not mask the interventions. We attempted to balance the risk of provider bias by providing detailed stability and failure criteria. In eight cases (five in "slow wean" group and three in "fast wean" group), infants were randomized prior to being ready to wean (NCPAP $>6$ CWP or not meeting the stability criteria). This protocol deviation could have increased the risk of provider bias, but one would expect that such bias would favor the "slow wean" arm as this is the standard practice at our institution.

Unexpectedly, a large proportion of infants in the "slow wean" arm did not receive antenatal steroids, which differs from standard obstetric practice at our institution. Given the high rates of antenatal steroids given in our institution, it is possible that this indicates more mothers in this group did not present to the hospital in time to receive steroids prior to delivery or that their delivery was emergent in nature. The difference in the rates of antenatal steroids between the two arms could be confounded by a difference in the severity of lung disease. However, as other 
indicators of lung disease such as duration of mechanical ventilation and NCPAP prior to randomization were not different between the two groups, we assume that this did not affect our outcomes.

\section{CONCLUSIONS}

Based on the results of our study, we conclude that it is possible that NCPAP 6CWP can be safely discontinued in premature infants who demonstrate respiratory stability without further weaning. As weaning pressures resulted in more days on NCPAP, prolonged weaning could result in unnecessary prolongation of NCPAP use. Because of the lack of prior studies evaluating this particular primary outcome, we chose to perform this study as a pilot trial without a prior sample size assessment. Therefore, these data should be confirmed by a larger trial with adequate power to determine differences in both primary and secondary outcomes.

\section{REFERENCES}

1. Mulrooney N, Champion Z, Moss TJ. Surfactant and physiologic responses of preterm lambs to continuous positive airway pressure. Am J Respir Crit Care Med. 2005;171:488-493.

2. Ho JJ, Subramaniam P, Henderson-Smart DJ, Davis PG. Continuous distending pressure for respiratory distress syndrome in preterm infants. Cochrane Database Syst Rev. 2008;10.

3. Morley $\mathrm{CJ}$, for the COIN Trial Investigators. Nasal CPAP or intubation at birth for very preterm infants. N Engl J Med. 2008;358:700-708.

4. Finer NN, for the SUPPORT Study Group of the Eunice Kennedy Shriver NICHD Neonatal Research Network. Early CPAP versus surfactant in extremely preterm infants. N Engl J Med. 2010;362:1970-1979.

5. Dunn MS, Kaempf J, de Klerk A, de Klerk R, Reilly M, Howard D. et al, Vermont Oxford Network DRM Study Group. Randomized trial comparing 3 approaches to the initial respiratory management of preterm neonates. Pediatrics. 2011;128: e1069-76.

6. Jobe $\mathrm{AH}$, Hillman N, Polglase G, Kramer BW, Kallapur S, Pillow J. Injury and inflammation from resuscitation of the preterm infant. Neonatology. 2008;94:190-96.

7. Foglia $E E$, Jensen $E A$, Kirpalani $H$. Delivery room interventions to prevent bronchopulmonary dysplasia in extremely preterm infants. J Perinatol. 2017;37:1171-79.
8. Abdel-Hady H, Mohareb S, Khashaba M, Abu-Alkhair M, Greisen G. Randomized controlled trial of disconnection of nasal CPAP in stable preterm infants breathing room air. Acta Pediatr. 1998;87:82-7.

9. Abdel-Hady $\mathrm{H}$, Shouman $\mathrm{B}$, Aly H. Early weaning from CPAP to high flow nasal cannula in preterm infants is associated with prolonged oxygen requirement: a randomized controlled trial. Early Hum Dev. 2011;87:205-8.

10. Todd DA, Wright A, Broom M, Chauhan M, Meskell S, Cameron C, et al. Methods of weaning preterm babies $<30$ weeks gestation off CPAP: a multicentre randomised controlled trial. Arch Dis Child Fetal Neonatal Ed. 2012;97:F236-F240.

11. Amatya $S$, Macomber M, Bhutada A, Rastogi $D$, Rastogi $S$. Sudden versus gradual pressure wean from Nasal CPAP in preterm infants: a randomized controlled trial. J Perinatol. 2017;37:662-67.

12. Jensen CF, Sellmer A, Ebbesen F, Cipliene R, Johansen A, Hansen RM, et al. Sudden vs pressure wean from nasal continuous positive airway pressure in infants born before 32 weeks of gestation: a Randomized Clinical Trial. JAMA Pediatr. 2018;172:824-831.

13. Amatya S, Rastogi D, Bhutada A, Rastogi S. Weaning of nasal CPAP in preterm infants: who, when and how? a systematic review of the literature. World J Pediatr. 2015;11:7-13.

14. Rastogi S, Wong W, Gupta A, Bhutada A, Maimonides Neonatal Group. Gradual versus sudden weaning from nasal CPAP in preterm infants: a pilot randomized controlled trial. Respir Care. 2013;58:511-516.

\section{ACKNOWLEDGEMENTS}

We thank the clinical faculty, nurse practitioners, fellows, residents, nurses, and respiratory therapists who made the implementation of our study possible. We would also like to thank the biostatisticians at the University of Texas Health Science Center - Houston for their assistance and review of our statistical analysis.

\section{COMPETING INTERESTS}

The authors declare no competing interests.

\section{ADDITIONAL INFORMATION}

Correspondence and requests for materials should be addressed to Brittany Duyka.

Reprints and permission information is available at http://www.nature.com/ reprints

Publisher's note Springer Nature remains neutral with regard to jurisdictional claims in published maps and institutional affiliations. 